Vol. 15, $\mathrm{N}^{2} 2, \mathrm{pp}, 141-151$, December 1996

Iniversidad Católica del Norte

Antolagastia - Shile

\title{
PRODUCTS OF POWER WEIGHTS AND $A_{p}$ WEIGHTS
}

\author{
Randy Combs \\ Wrst Trxas A G M. University, Canyon, TX, U. S. A.
}

\begin{abstract}
Sufficient conditions are given for a product of an. $A_{p}$ weight and. a power weight to belong to the $A_{7}$ class of weights. These are gencralizations to $\mathbf{R}^{n}$ of $\mathbf{R}$ results given by Muckenhoupt, Whecdon, and Yung.
\end{abstract}




\section{Introduction}

Let $u$ be a measurable, nonnegative function on $\mathbf{R}^{n}$. The function $u$ is said to belong to the $A_{p}$ class of weight functions, $1<p<\infty$, if there is a constant $C$ such that

$$
\left(\frac{1}{|Q|} \int_{Q} u(x) d x\right)\left(\frac{1}{|Q|} \int_{Q} u(x)^{-1 /(p-1)} d x\right)^{p-1} \leq(:
$$

for all cubes $Q \subset \mathbf{R}^{n}$. When $p=1, u \in A_{1}$ if $u^{*}(x) \leq C u(x)$, where $u *$ is the Hardy-Littlewood maximal function of $u$ defined as

$$
u^{*}(x)=\sup _{Q ; x \in Q} \frac{1}{|Q|} \int_{Q}|u(y) \cdot| d y
$$

$A_{\infty}$ will denote the union $\cup_{p \geq 1} A_{p}$.

Among the many results concerning $A_{p}$ weights, we note the following two.

Let $u(Q)$ denote the weighted measure of $Q$ as follows:

$$
u(Q)=\int_{Q} u(x) d x
$$

If $2 Q$ denotes the cube with the same center as $Q$ but having twice the side length of $Q$ and there is a constant $C$ independent of $Q$ such that $u(2 Q) \leq C u(Q)$, we say that $u$ is a doubling weight, or satisfies a doubling condition. If $u \in A_{p}$, then it satisfies a doubling condition.

Let $r>1$. The function $u$ is said to satisfy the reverse Hölder condition, $R H_{r}$, if there exists a constant $C$ such that for every cube $Q$,

$$
\left(\frac{1}{|Q|} \int_{Q} u(x)^{r} d x\right)^{1 / r} \leq \frac{C}{|Q|} \int_{Q} u(x) d x .
$$

An equivalent condition for $u \in A_{\infty}$ is for $u$ to satisfy an $R H_{r}$ for some $r>1$.

Other results and historical remarks concerning $A_{p}$ weights may be found in [2]. The original results of this paper may be found in the author's $\mathrm{Ph} . \mathrm{D}$. dissertation [4]. The author would like to express his gratitude to his advisor, Douglas Kurtz, for the support and assistance given during the writing of the dissertation.

In this paper we find sufficient conditions, with $u \in A_{p}$, on the function

$$
g(x)=(1+|x|)^{l_{0}} \prod_{j=1}^{J}\left|x-p_{j}\right|^{l}
$$


for $g^{p} u \in A_{\infty}$. These results are higher dimensional generalizations of those found in [1]. The main result of this paper is the following theorem.

Theorem 1.1. Let $A>0$ and $t$ be in $\mathbf{R}^{n}$. Suppose that $h(x)$ is one of the functions $|x-t|, A+|x-t|$, or $|x-t| /(A+|x-t|)$. Let $v(x)$ be a function belonging to $A_{p}$ such that $v(x)^{1-p^{\prime}} \in R H_{r}$ where $r>1$. If $E$ satsifies $0 \leq E<n(p-1)(1-1 / r)$, then $h(x)^{E} v(x) \in A_{p}$.

As a consequence of this result, we have the following corollary.

Corollary 1.2. Suppose $1<p<\infty$. Let $N$ be an integer such that $N \geq-1$ and for $j=1, \ldots, J$, let $m_{j}$ be a nonnegative integer. Set $M=$ $\sum_{j=1}^{J} m_{j}-1$ and

$$
g(x)=(1+|x|)^{N-M} \prod_{j=1}^{J}\left|x-p_{j}\right|^{m_{j}} .
$$

If $v(x) \in A_{p}$, then $g(x)^{p} v(x) \in A_{\infty}$.

We fix the following notation. If $u$ and $v$ are functions on $\mathbf{R}^{n}$ and there are constants $a$ and $b$ independent of $x$ such that $a u(x) \leq v(x) \leq b u(x)$, then we write $u(x) \sim v(x)$.

The n-dimensional measure of a subset $S$ of $\mathbf{R}^{n}$ will be denoted by $|S|$, and a ball centered at $t \in \mathbf{R}^{n}$ with radius $r$ will be denoted by $B(t, r)$.

\section{Main Results}

Lemma 2.1. Suppose $R$ is a positive real number and $t$ is in $\mathbf{R}^{n}$. Let $u$ and $v$ be doubling weights such that $u(x) \leq C^{\prime} v(x)$ for $x$ belonging to the annulus $\{x: R<|x-t|<2 R\}$. Let $Q$ be a cube contained in the ball $B(t, 2 R)$. If $Q$ intersects $\{x: R<|x-t|<2 R\}$, then

$$
u(Q) \leq C v(Q)
$$

for some constant $C$ independent of $Q$.

\section{Proof:}

Let $Q$ be a cube satisfying the hypothesis. If $Q \subset\{x: R<|x-t|<2 R\}$, then the conclusion holds by hypothesis. We can reduce to the case where $Q$ is contained in the annulus by choosing an appropriate cube $Q_{1}$ that is 
a subset of the annulus and whose weighted measure compares nicely with $Q$ through the doubling property.

Note that $Q$ will have at least one diagonal intersecting the annulus. If the intersection consists of two parts, we let $\tilde{\tilde{a}}$ be the longer piece. We denote by $D$ a diagonal of $Q$ that yields the longest $\tilde{D}$. If there is more than one diagonal satisfying this property, fix one of them and call it $D$.

The following two cases are considered, depending on the length of $\tilde{D}$ :

$$
\begin{aligned}
& \operatorname{len}(\tilde{D}) \geq \frac{R}{2} \\
& \operatorname{len}(\widetilde{D})<\frac{R}{2}
\end{aligned}
$$

Suppose $D$ satisfies $(2.2)$. Extend $\tilde{D}$ to the boundary of $B(t, 2 R)$ and let $Q_{1}$ be the cube having this extension of $\tilde{D}$ as a diagonal. We denote this diagonal by $D_{1}$.

We now compare the weighted measure of $Q$ and $Q_{1}$. The first step is to show that $Q_{1}$ is contained in $4 \sqrt{n} Q$.

The inequality $(2.2)$ and the hypothesis imply that $R / 2 \leq \operatorname{len}(\mathrm{D}) \leq 4 R$. Thus, the side length of $Q$ satisfies

$$
\frac{R}{2 \sqrt{n}} \leq|Q|^{1 / n} \leq \frac{2 R}{\sqrt{n}}
$$

Also, the diagonal $D_{1}$ satsifies $R \leq \operatorname{len}\left(D_{1}\right) \leq 2 R$, so that for the side length of $Q_{1}$ we have

$$
\frac{R}{\sqrt{n}} \leq\left|Q_{1}\right|^{1 / n} \leq \frac{2 R}{\sqrt{n}}
$$

It follows that $Q_{1} \subset 4 \sqrt{n} Q$.

Since $|4 \sqrt{n} Q| \leq(8 R)^{n}$ and $\left|Q_{1}\right| \geq R^{n}$, and by the doubling property of $u$, we have

$$
u(Q) \leq u(4 \sqrt{n Q} Q) \leq C u\left(Q_{1}\right) .
$$

But since $Q_{1} \subset\{x: R<|x-t|<2 R\}$ and $v$ is a doubling measure, we have

$$
C u\left(Q_{1}\right) \leq C v\left(Q_{1}\right) \leq C v(4 \sqrt{ } n Q) \leq C v(Q) .
$$

Thus, if $D$ satisfies the condition in $(2.2)$, then $u(Q) \leq C v(Q)$. 
Now suppose that $D$ satisfies the condition in (2.3). We want to compare $Q$ with a cube $Q_{1}$ contained in the annulus as we did for (2.2). Since we have no control on the size of $Q$, we cannot perform the same construction. However, we will dilate the cube far enough into the amnulus to obtain a sub-cube of the dilated cube that will also be contained in the anmulus. The sub-cube will have measure comparable to the measure of $Q$.

We dilate $Q$ by the factor $(5 / 4) \sqrt{n}$. The length of $D$ can be no longer than $4 R$ by hypothesis and (2.2). The resulting dilation of $D,(5 / 4) D$, is obtained by adding on a line segment with length len(D/8) to both encts of $D$. We have

$$
\operatorname{len}\left(\left(\frac{5}{4}\right) D\right) \leq \frac{R}{2}+\left(\frac{3}{8}\right) R=\left(\frac{7}{8}\right) R .
$$

Thus, $(5 / 4) \sqrt{n} Q$ is a subset of $\{x:|x-t|<2 R\}$.

Let $Q_{1}$ be the cube that has for one of its diagonals the line segment in the annulus that was added to $D$ in the dilation of $Q$. Note that | $Q_{1}\left|=\left(1 / 8^{n}\right)\right| Q \mid$. By the doubling properties of $u$ and $v$ and since $Q_{1} \subset\{x: R<|x-t|<2 R\}$, we have

$$
u(Q) \leq C u\left(Q_{1}\right) \leq C v\left(Q_{1}\right) \leq C v(Q) .
$$

Therefore, if $D$ satisfies (2.2), then $u(Q) \leq C v(Q)$.

This concludes the proof of the Lemma.

Lemma 2.2 will enable us to deduce conditions on the exponents appearing in the function

$$
(1+|x|)^{l_{0}} \prod_{j=1}^{J}\left|x-p_{j}\right|^{l_{j}}
$$

that will put $g$ in $A_{p}$. These conditions are made explicit in Corollary 2.3. Lemma 2.2 appears in [2] in the case where $n=1$.

Lemma 2.2. Suppose $R>0, t$ is in $\mathbf{R}^{n}$, and $1<p<\infty$. Let u and $v$ be functions in $A_{p}$ such that $u(x) \sim v(x)$ for $R<|x-t|<2 R$. If $w$ is a nonnegative function such that $w(x) \sim u(x)$ for $\mathrm{x}$ in $B(t, R)$ and $w(x) \sim v(x)$ for $\mathrm{x}$ in $B(t, R)^{c}$, then $w$ is in $A_{p}$.

\section{Proof:}

Let $Q$ be a cube in $\mathbf{R}^{n}$. The hypothesis implies that if $x \in B(t, 2 R)$, we have $w(x) \sim u(x)$. Consequently, if $Q$ is contained in $B(t, 2 R)$ or in $B(t, R)^{r}$, the $A_{p}$ condition is satisfied trivially. Therefore, we assume that $Q$ intersects $B(t, R)$ and $B(t, 2 R)^{c}$. 
Suppose $Q$ has one vertex in the ball $B(t, R)$. Let $D$ be the diagonal emanating from that vertex and let $Q_{1}$ be the cube that has for one of its diagonals the line segment $D \cap B(t, 2 R)$. We have for this case

$$
\begin{gathered}
\int_{Q} w(x) d x \leq C\left(\int_{Q \cap B(t, R)} u(x) d x+\int_{Q \cap B(t, R)^{c}} v(x) d x\right) \\
\leq C \int_{Q_{1}} u(x) d x+\int_{Q} v(x) d x \\
\leq C \int_{Q} v(x) d x .
\end{gathered}
$$

The last inequality follows from Lemma 2.1.

We also do the same for $w(x)^{1-p^{\prime}}$. We can apply Lemma 2.1 in this case since $u^{1-p^{\prime}}$ and $v^{1-p^{\prime}}$ are in $A_{p^{\prime}}, w(x) \sim v(x)$ for $x$ in $B(t, R)^{c}$, and $u(x) \sim v(x)$ in the annulus. Thus, we have

$$
\int_{Q} w(x)^{1-p^{\prime}} d x \leq C \int_{Q} v(x)^{1-p^{\prime}} d x .
$$

When we combine this with the above, we see that the $A_{p}$ condition is satisfied for cubes that have a vertex in the ball $B(t, R)$.

Now suppose $Q$ intersects $B(t, R)$ and $B(t, 2 R)^{c}$. We subdivide $Q$ finely enough into subcubes $Q_{i}$ that have at most one vertex in $B(t, R)$.

We have

$$
\int_{Q} w(x) d x \leq \sum_{Q_{i} \cap B(t, R) \neq \phi} \int_{Q_{i}} w(x) d x+\sum_{Q_{i} \cap B(t, R)=\phi} \int_{Q_{i}} w(x) d x .
$$

By hypothesis, the second sum is bounded by $C v(Q)$, and for the first sum, we have

$$
\sum_{Q_{i} \cap B(t, R) \neq \phi} \int_{Q_{i}} w(x) d x \leq \sum_{Q_{i} \subset B(t, 2 R)} \int_{Q_{i}} u(x) d x+\sum_{Q_{i} \cap B(t, 2 R)^{\mathrm{c}} \neq \phi} \int_{Q_{i}} w(x) d x .
$$

which is also bounded by $C v(Q)$ by Lemma 2.1 and (2.3).

When we repeat the process for $w(x)^{1-p^{\prime}}$, we obtain the $A_{p}$ condition for the general case. This concludes the proof of the Lemma.

The following corollary gives us necessary and sufficient conditions on the exponents appearing in the function $g$ defined in (1.1) for $g$ to be in $A_{p}$. 
Corollary 2.3. Let $1<p<\infty$. Given real numbers $l_{j}, j=0,1, \ldots, J$, set

$$
g(x)=(1+|x|)^{l_{0}} \prod_{j=1}^{J}\left|x-p_{j}\right|^{l},
$$

with $p_{j} \in \mathbf{R}^{n}$. The function $g$ belongs to $A_{p}$ if and only if

$$
-n<l_{j}<n(p-1)
$$

for $j=1, \ldots, J$, and

$$
-n<l_{0}+\sum_{j=1}^{J} l_{j}<n(p-1)
$$

Proof:

We will show that (2.4) and (2.5) are sufficient for $g$ to be in $A_{p}$. The proof of the necessity is similar.

We proceed by induction and apply Lemma 2.2 when $J=0,1$ and at the inductive step.

We consider first the case where $J=0$. We have for $x$ in the ball $B(0,1)$

$$
(1+|x|)^{l_{0}} \sim 1
$$

and for $x$ outside the ball $B(0,1)$

$$
(1+|x|)^{l_{0}} \sim|x|^{l_{0}}
$$

with the function on the right in $A_{p}$ by hypothesis. Furthermore, we have $|x| \sim 1$ for $x$ in the annulus $\{x: 1<|x|<2\}$. Thus, Lemma 2.2 implies that $(1+|x|)^{l_{0}} \in A_{p}$. In a similar manner, the $J=1$ follows from Lemma 2.2 with $R=2\left(\left|p_{1}\right|+1\right)$.

Now suppose that for each $J \leq N-1$, we have $g(x) \in A_{p}$ if (2.4) and (2.5) are met. Let $p_{j}, j=1, \ldots N$, be in $\mathbf{R}^{n}$ and $l_{j}, j=0, \ldots N$ be real numbers satisfying (2.4) and (2.5). Set $d=\min _{i \neq j}\left|p_{i}-p_{j}\right|$ if $N>1$ and $d=\left|p_{1}\right|$ if $N=1$.

Since $(1+|x|) \sim\left|x-p_{j}\right|, j \neq N$, for $x \in B\left(p_{N}, d / 4\right)$, we have

$$
(1+|x|)^{l_{0}} \prod_{j=1}^{N}\left|x-p_{j}\right|^{l_{3}} \sim(1+|x|)^{l_{0}+\sum_{j=1}^{N-1} l_{j}}\left|x-p_{N}\right|^{l_{N}} .
$$

Note that by the induction hypothesis, the right hand side is in $A_{p}$. 
We have $(1+|x|) \sim\left|x-p_{N}\right|$ for $x \in B\left(p_{N}, d / 4\right)^{c}$. Thus,

$$
(1+|x|)^{l_{0}} \prod_{j=1}^{N}\left|x-p_{j}\right|^{l_{j}} \sim(1+|x|)^{l_{0}+l_{N}} \prod_{j=1}^{N-1}\left|x-p_{j}\right|^{l_{j}}
$$

and the function on the right is in $A_{p}$ by the induction hypothesis. Moreover, for $x$ such that $d / 4<\left|x-p_{N}\right|<d / 2$, we have

$$
(1+|x|)^{l_{0}+l_{N}} \prod_{j=1}^{N-1}\left|x-p_{j}\right|^{l_{3}} \sim(1+|x|)^{l_{0}+\sum_{j=1}^{N-1} l_{j}}\left|x-p_{N}\right|^{l_{N}} .
$$

Thus, the hypothesis of Lemma 2.2 is met and hence,

$$
(1+|x|)^{l_{0}} \prod_{j=1}^{J}\left|x-p_{j}\right|^{l_{j}} \in A_{p} .
$$

This concludes the proof of the corollary.

The following theorem appears in [1] in the case where $n=1$ and the proof given here is a straightforward generalization that is included for completeness. From the theorem, we obtain a corollary giving sufficient conditions on the exponents of the function $g$ in (1.1) for $g^{p} v$ to belong to $A_{\infty}$.

Theorem 1.1. Let $A>0$ and $t$ be in $\mathbf{R}^{n}$. Suppose that $h(x)$ is one of the functions $|x-t|, A+|x-t|$, or $|x-t| /(A+|x-t|)$. Let $v(x)$ be a function belonging to $A_{p}$ such that $v(x)^{1-p^{\prime}} \in R H_{r}$ where $r>1$. If $E$ satsifies $0 \leq E<n(p-1)(1-1 / r)$, then $h(x)^{E} v(x) \in A_{p}$.

\section{Proof:}

We prove the case where $h(x)=|x-t|$. The other two cases will follow from Lemma 2.2 .

Let $Q$ be a cube. We first sub-divide $Q$ into $4^{n}$ sub-cubes. Next we choose the sub-cube that is furthest in distance from $t$, denote it by $Q^{\prime}$, and set $d=\max _{x \in Q^{\prime}}|x-t|$. We note that $|x-t| \sim d$ for $x \in Q^{\prime}$ and $|x-t| \leq d$ for $x \in Q$.

As in the previous proofs, we want to compare the weighted measure of $Q$ and $Q^{\prime}$. The weight function in this case is $w(x)=|x-t|^{\left(r^{\prime} E\right) /(1-p)}$ where $E$ is a number satisfying

$$
\frac{r^{\prime} E}{p-1}<n
$$


If $E$ satisfies the inequality, then the function $w(x)$ is an $A_{1}$ weight and thus is a doubling weight.

We show that $|x-t|^{E} v(x)$ is in $A_{p}$ for this choice of $E$. By Hölder's inequality, the doubling property, and $|x-t| \sim d$ for $x \in Q^{\prime}$, we have

$$
\int_{Q}\left(|x-t|^{E} v(x)\right)^{1-p^{\prime}} d x \leq C\left(\int_{Q} v(x)^{\left(1-p^{\prime}\right) r} d x\right)^{1 / r} d^{E /(1-p)}|Q|^{1 / r^{\prime}}
$$

and from the hypothesis that $v(x)^{1-p^{\prime}} \in R H_{r}$, the right hand side is

$$
\leq C d^{-E /(p-1)} \int_{Q} v(x)^{\left(1-p^{\prime}\right)} d x .
$$

Since $E \geq 0$ and $|x-t| \leq d$, we have

$$
\begin{gathered}
\frac{1}{|Q|} \int_{Q}|x-t|^{E} v(x) d x\left(\frac{1}{|Q|} \int_{Q}\left(|x-t|^{E} v(x)\right)^{1-p^{\prime}} d x\right)^{p-1} \\
\leq C \frac{1}{|Q|} \int_{Q} v(x) d x \\
\left(\frac{1}{|Q|} \int_{Q} v(x)^{1-p^{\prime}} d x\right)^{p-1} \\
\leq C .
\end{gathered}
$$

The last inequality follows since $v(x) \in A_{p}$. This concludes the proof of the Theorem. $\square$

Corollary 1.2. Suppose $1<p<\infty$. Let $N$ be an integer such that $N \geq-1$ and for $j=1, \ldots, J$, let $m_{j}$ be a nonnegative integer. Set $M=$ $\sum_{j=1}^{J} m_{j}-1$ and

$$
g(x)=(1+|x|)^{N-M} \prod_{j=1}^{J}\left|x-p_{j}\right|^{m_{j}} .
$$

If $v(x) \in A_{p}$, then $g(x)^{p} v(x) \in A_{\infty}$.

\section{Proof:}

The proof proceeds by induction on $J$.

We consider the case where $N \geq-1$ and $J=0$. We have $v(x)^{1-p^{\prime}} \in$ $A_{p^{\prime}} \cap R H_{z}$ for some $z>1$. We choose by Theorem 1.1 a $q \geq p$ such that

$$
(N+1) p<n(q-1)\left(1-\frac{1}{z}\right) .
$$


If $v^{1-q^{\prime}} \in A_{q^{\prime}} \cap R H_{z}$, then Theorem 1.1 implies the conclusion of the Corollary. Furthermore, from results in [3], this is equivalent to $v^{z\left(1-q^{\prime}\right)} \in$ $A_{\infty}$ and $v \in A_{\infty}$.

We have by hypothesis $v \in A_{\infty}$. We now show that $\left.v^{z(1} q^{\prime}\right) \in A_{\infty}$. Since $q>p$, we have $p^{\prime}>q^{\prime}$. Set $\varepsilon=\left(q^{\prime}-1\right) /\left(p^{\prime}-1\right)$ and note that $0<\varepsilon<1$. Since $v^{z\left(1-p^{\prime}\right)} \in A_{\infty}$, there is a $P$ such that $v^{z\left(1-p^{\prime}\right)} \in A_{P}$, which, from results in [2], implies

$$
v^{z\left(1-q^{\prime}\right)}=v^{\varepsilon z\left(1-p^{\prime}\right)} \in A_{\varepsilon P+1-\varepsilon} .
$$

We conclude $v^{z\left(1-q^{\prime}\right)} \in A_{\infty}$.

For the inductive step, we assume for some $P \geq p$,

$$
(1+|x|)^{p(N-M)} \prod_{j=1}^{J-1}\left|x-p_{j}\right|^{p m_{j}} v(x) \in A_{P} .
$$

where $M=\sum_{j=1}^{J-1} m_{j}-1$.

Let $p_{J} \in \mathbf{R}^{n}$ and $m_{J}$ be a nonnegative integer. We apply the same argument above to the factor $\left|x-p_{j}\right|^{m J}$ and the function

$$
(1+|x|)^{p(N-M)} \prod_{j=1}^{J-1}\left|x-p_{j}\right|^{p r n_{j}} v(x)
$$

to obtain

$$
(1+|x|)^{p\left(N-M-m_{J}\right)} \prod_{j=1}^{J}\left|x-p_{j}\right|^{p m_{j}} v(x) \in A_{\infty} .
$$

This concludes the proof of the corollary. $\square$ 


\section{References}

[1] B. Mukenhoups, R. L. Wheeden, and W. Young, Sufficiency conditions for $L^{p}$ multipliers with general weights, Trans. Amer. Math. Soc., 300 , pp. $4633-503,(1987)$.

[2] J. García - Cuerva and J. L. Rubio de Francia, Weighted Norm Inequalities and Related Topics, Nortl - Holland, Amsterdam (1985).

[3] J. - O. Strömberg and A. Torchinsky, Weighted Hardy Spaces, Lecture Notes and Related Topics, North - Holland, Amsterdam (1985).

[4] R. Combs, Ph. D. Dissertation, New Mexico State University (1991).

Received : April 30, 1996.

\section{Randy Combs}

Department of Mathematics, Physical Sciences, and Engineering Technology

West Texas A \& M. University

Canyon, TX

U. S. A. 